\title{
Numerical Approach Based on Two-Dimensional Fractional-Order Legendre Functions for Solving Fractional Differential Equations
}

\author{
Qingxue Huang, ${ }^{1,2,3}$ Fuqiang Zhao, ${ }^{1,3}$ Jiaquan Xie, ${ }^{1,3}$ Lifeng Ma, ${ }^{1,3}$ \\ Jianmei Wang, ${ }^{1,3}$ and Yugui $\mathrm{Li}^{1,3}$ \\ ${ }^{1}$ College of Mechanical Engineering, Taiyuan University of Science \& Technology, Taiyuan, Shanxi 030024, China \\ ${ }^{2}$ College of Mechanical Engineering, Taiyuan University of Technology, Taiyuan, Shanxi 030024, China \\ ${ }^{3}$ Shanxi Provincial Key Laboratory of Metallurgical Device Design Theory and Technology, Taiyuan, Shanxi 030024, China \\ Correspondence should be addressed to Fuqiang Zhao; zfqgear@163.com
}

Received 23 August 2016; Revised 2 December 2016; Accepted 18 December 2016; Published 23 January 2017

Academic Editor: Vincenzo Scalzo

Copyright (C) 2017 Qingxue Huang et al. This is an open access article distributed under the Creative Commons Attribution License, which permits unrestricted use, distribution, and reproduction in any medium, provided the original work is properly cited.

In this paper, a robust, effective, and accurate numerical approach is proposed to obtain the numerical solution of fractional differential equations. The principal characteristic of the approach is the new orthogonal functions based on shifted Legendre polynomials to the fractional calculus. Also the fractional differential operational matrix is driven. Then the matrix with the Tau method is utilized to transform this problem into a system of linear algebraic equations. By solving the linear algebraic equations, the numerical solution is obtained. The approach is tested via some examples. It is shown that the FLF yields better results. Finally, error analysis shows that the algorithm is convergent.

\section{Introduction}

Fractional-order calculus has a long history. Compared with integer order, fractional differential equations show many advantages over the simulation of physical phenomena [1]. In recent decades, fractional partial differential equations (FPDEs) [2] are widely used to describe engineering processes and dynamical systems; more and more researchers have devoted to study a variety of methods for solving fractional differential equations. The most commonly used methods are generalized differential transform method [3], Adomian decomposition method [4], finite difference method [5], and so on. Recently, the authors have proposed wavelet methods for solving a class of fractional convectiondiffusion equations. In [6], different families of wavelets [711] have been widely applied for solving problems of partial differential equations. In [12], the authors have obtained the approximate solutions of fractional differential equations using the generalized block pulse operational matrix.

The methods based on the orthogonal functions [13-15] are powerful and wonderful for solving FPDEs and have achieved great success in this field. Now the operational matrices of fractional derivative and integral [14-16] for Bernstein polynomials and Jacobi polynomials [15] have been derived. However, these polynomials use integer power series to approximate fractional ones; it cannot accurately represent properties of fractional integral and differential. Recently, in [17], Rida and Yousef have proposed a fractional extension of classical Legendre polynomials by replacing the integer order derivative in Rodrigues formula [18] with fractional-order derivatives. These functions are complex, so they are not unsuitable for solving FPDEs. Subsequently, Kazem et al. put forward the orthogonal fractional-order Legendre functions based on shifted Legendre polynomials. In [19], they used these functions to obtain the numerical solutions of fractional partial differential equations. Numerical results show that their methods are effective, accurate, and easy to implement. In this paper, FLF is suitable to characterize the properties of fractional partial differential equations; thus we attempt to use fractional-order Legendre functions to acquire numerical solution of the FPDEs.

The article is organized as follows. In Section 2, we introduce some basic definitions of fractional derivatives and integrals. In Section 3, we construct the FLFs and introduce 
some properties. In Section 4, the fractional operational matrices of FLFs are obtained. In Section 5, we use the proposed method to solve FPDEs. In Section 6, the proposed method is tested through several numerical examples. In Section 7, the error analysis is given. Finally Section 8 concludes the paper.

\section{Preliminaries and Notations}

In this section, we recall the essentials of the fractional calculus theory that will be used in this article.

Definition 1. The Riemann-Liouville fractional integral operator of order $\alpha>0$ is defined as

$$
\begin{aligned}
I^{\alpha} u(t) & =\frac{1}{\Gamma(\alpha)} \int_{0}^{t}(t-s)^{\alpha-1} u(s) d s, \quad \alpha>0, \\
I^{0} u(t) & =u(t) .
\end{aligned}
$$

Definition 2. The Riemann-Liouville definition of fractional differential operator is given as follows:

$D_{*}^{\alpha} u(t)$

$$
= \begin{cases}\frac{1}{\Gamma(m-\alpha)} \frac{d^{m}}{d t^{m}} \int_{0}^{t} \frac{u(s)}{(t-s)^{\alpha-m+1}} d s, & \alpha>0, m-1 \leq \alpha<m, \\ \frac{d^{m} u(t)}{d t^{m}}, & \alpha=m .\end{cases}
$$

(i) $D^{\alpha} C=0$,

(ii) $\quad D^{\alpha} t^{v}= \begin{cases}0, & \text { for } v \in N_{0}, v<\lceil\alpha\rceil, \\ \frac{\Gamma(v+1)}{\Gamma(v+1-\alpha)} t^{v-\alpha}, & \text { for } v \in N_{0}, \quad v \geq\lceil\alpha\rceil \text { or } v \notin N_{0}, v>\lfloor\alpha\rfloor .\end{cases}$

(iii) $D^{\alpha}\left(\sum_{i=0}^{m} c_{i} u_{i}(t)\right)=\sum_{i=0}^{m} c_{i} D^{\alpha} u_{i}(t)$, where $\left\{c_{i}\right\}_{i=0}^{m}$ are constants.
Definition 3. The Caputo definition of fractional differential operator is defined as

$$
\begin{aligned}
& D^{\alpha} u(t) \\
& \quad= \begin{cases}\frac{1}{\Gamma(m-\alpha)} \int_{0}^{t} \frac{u^{(m)}(\tau)}{(t-\tau)^{\alpha-m+1}} d \tau, & m-1 \leq \alpha<m, \\
\frac{d^{m} u(t)}{d t^{m}}, & \alpha=m .\end{cases}
\end{aligned}
$$

The relation between the Riemann-Liouville operator and Caputo operator is given by the following expressions:

$$
\begin{aligned}
& D^{\alpha} I^{\alpha} u(t)=u(t) \\
& I^{\alpha} D^{\alpha} u(t)=u(t)-\sum_{k=0}^{m-1} u^{(k)}\left(0^{+}\right) \frac{t^{k}}{k !} .
\end{aligned}
$$

For $\alpha \geq 0, v \geq-1$, and constant $C$, Caputo fractional derivative has some basic properties which are needed here as follows:
Definition 4 (generalized Taylor's formula). Suppose that $D^{i \alpha} u(t) \in C[0,1]$ for $i=0,1, \ldots, m-1$, then one has

$$
\begin{aligned}
u(t)= & \sum_{i=0}^{m-1} \frac{t^{i \alpha}}{\Gamma(i \alpha+1)} D^{i \alpha} u\left(0^{+}\right) \\
& +\frac{t^{m \alpha}}{\Gamma(m \alpha+1)} D^{m \alpha} u(\xi),
\end{aligned}
$$

where $0<\xi \leq t, \forall t \in[0,1]$. Also, one has

$$
\left|u(t)-\sum_{i=0}^{m-1} \frac{t^{i \alpha}}{\Gamma(i \alpha+1)} D^{i \alpha} u\left(0^{+}\right)\right| \leq M_{\alpha} \frac{t^{m \alpha}}{\Gamma(m \alpha+1)},
$$

where $M_{\alpha} \geq\left|D^{m \alpha} u(\xi)\right|$.

In case $\alpha=1$, the generalized Taylor's formula (6) is the classical Taylors formula.

\section{Definitions of Fractional-Order Legendre Definition and Function Approximation}

3.1. Shifted Legendre Polynomials. The well-known Legendre polynomials are orthogonal with the weight function $\omega(x)=$ 1 , which is defined on the interval $[-1,1]$; namely,

$$
\int_{-1}^{1} P_{m}(x) P_{n}(x) \omega(x) d x= \begin{cases}\frac{2}{(2 n+1)}, & m=n \\ 0, & m \neq n .\end{cases}
$$

In order to use these polynomials on the interval $[0,1]$, let $t=2 x-1$, and then we derived the shifted Legendre polynomials $P_{n}(2 x-1)$ which are denoted by $L_{n}(t)$. The 
shifted Legendre polynomials are orthogonal with the weight function $\omega_{l}(t)=1$ over $[0,1]$, with the orthogonally property

$$
\int_{0}^{1} L_{i}(t) L_{j}(t) \omega_{l}(t) d t= \begin{cases}0, & i \neq j \\ \frac{1}{2 i+1}, & i=j .\end{cases}
$$

Then $L_{i}(t)$ can be obtained as follows:

$$
\begin{aligned}
L_{i+1}(t) & =\frac{(2 i+1)}{i+1} L_{i}(t)-\frac{i}{i+1} L_{i-1}(t), \\
& \\
L_{0}(t) & =1, \\
L_{1}(t) & =2 t-1,2, \ldots,
\end{aligned}
$$

The analytic form of the shifted Legendre polynomial $L_{n}(t)$ of degree $n$ is given by

$$
\begin{aligned}
& L_{n}(t)=\sum_{i=0}^{n} b_{n i} t^{i}, \\
& L_{n}(0)=(-1)^{n}, \\
& L_{n}(1)=1,
\end{aligned}
$$

where $b_{n i}=(-1)^{n+i}\left((n+i) ! /(n-i) !(i !)^{2}\right)$.

3.2. Fractional-Order Legendre Definition. We define the fractional-order Legendre functions (FLFs) by introducing the change of variable $t=x^{\alpha}$ and $\alpha>0$ on shifted Legendre polynomials. The fractional-order Legendre function $L_{i}\left(x^{\alpha}\right)$ is denoted by $F l_{i}^{\alpha}(x)$. The fractional-order Legendre functions are a particular solution of the normalized eigenfunctions of the singular Sturm-Liouville problem.

$$
\begin{aligned}
\left(\left(x-x^{1+\alpha}\right) F l_{i}^{\prime \alpha}(x)\right)^{\prime}+\alpha^{2} i(i+1) x^{\alpha-1} F l_{i}(x) & =0, \\
x & \in[0,1] .
\end{aligned}
$$

Then $F l_{i}^{\alpha}(x)$ can be obtained as follows:

$$
\begin{aligned}
F l_{i+1}^{\alpha}(x) & =\frac{(2 i+1)\left(2 x^{\alpha}-1\right)}{i+1} F l_{i}^{\alpha}(x)-\frac{i}{i+1} F l_{i-1}^{\alpha}(x), \\
i=1,2, \ldots, & \\
F l_{0}^{\alpha}(x) & =1, \\
F l_{1}^{\alpha}(x) & =2 x^{\alpha}-1 .
\end{aligned}
$$
follows:

We can derive the analytic form of $F l_{i}^{\alpha}(x)$ of degree $i \alpha$ as

$$
F l_{i}^{\alpha}(x)=\sum_{s=0}^{i} b_{s, i} x^{s \alpha}
$$

where $b_{s, i}=(-1)^{i+s}(i+s) ! /(i-s) !(s !)^{2}$ and $F l_{i}^{\alpha}(0)=$ $(-1)^{i}, F l_{i}^{\alpha}(1)=1$.
Theorem 5. The FLFs are orthogonal with the weight function $\omega_{l}^{\alpha}(x)=x^{\alpha-1}$ on the interval $[0,1]$; then the orthogonally condition is

$$
\int_{0}^{1} F l_{n}^{\alpha}(x) F l_{m}^{\alpha}(x) \omega_{l}^{\alpha}(x) d x=\frac{1}{(2 n+1) \alpha} \delta_{n m} .
$$

Proof. With $\int_{0}^{1} L_{n}(t) L_{m}(t) \omega_{l}(t) d t=(1 /(2 i+1)) \delta_{n m}$, where $\delta_{n m}$ is the Kronecker function, let $t=x^{\alpha}$, and then we have

$$
\begin{aligned}
& \int_{0}^{1} L_{n}(t) L_{m}(t) d t=\int_{0}^{1} L_{n}\left(x^{\alpha}\right) L_{m}\left(x^{\alpha}\right) \alpha x^{\alpha-1} d x \\
& =\frac{1}{2 n+1} \delta_{n m}, \\
& \int_{0}^{1} L_{n}\left(x^{\alpha}\right) L_{m}\left(x^{\alpha}\right) \alpha x^{\alpha-1} d x \\
& =\int_{0}^{1} F l_{n}^{\alpha}(x) F l_{m}^{\alpha}(x) \alpha x^{\alpha-1} d x=\frac{1}{2 n+1} \delta_{n m}, \\
& \int_{0}^{1} F l_{n}^{\alpha}(x) F l_{m}^{\alpha}(x) x^{\alpha-1} d x=\frac{1}{(2 n+1) \alpha} \delta_{n m} .
\end{aligned}
$$

3.3. Function Approximation. Suppose $u(x) \in L^{2}[0,1]$; it can be expanded in terms of the fractional-order Legendre functions as follows:

$$
u(x)=\sum_{i=0}^{\infty} c_{i} F l_{i}^{\alpha}(x),
$$

where the coefficients $c_{i}$ are obtained by

$$
\begin{aligned}
& c_{i}=\alpha(2 i+1) \int_{0}^{1} F l_{i}^{\alpha}(x) u(x) \omega_{l}^{\alpha}(x) d x, \\
& i=0,1,2, \ldots .
\end{aligned}
$$

If we consider truncated series in (17), we obtain

$$
u(x) \approx u_{m}(x)=\sum_{i=0}^{m-1} c_{i} F l_{i}^{\alpha}(x)=\mathbf{C}^{T} \boldsymbol{\Phi}(x),
$$

where $\mathbf{C}$ and $\boldsymbol{\Phi}(x)$ are $m \times 1$ vector given by

$$
\begin{aligned}
\mathbf{C} & =\left[c_{0}, c_{1}, \ldots, c_{m-1}\right]^{T}, \\
\Phi(x) & =\left[F l_{0}^{\alpha}(x), F l_{1}^{\alpha}(x), \ldots, F l_{m-1}^{\alpha}(x)\right]^{T} .
\end{aligned}
$$

Theorem 6. Suppose $D^{i \alpha} u(x) \in C[0,1]$ for $i=0,1,2, \ldots, m-$ 1. $(2 m+1) \alpha \geq 1$ and $\mathbf{P}_{m}^{\alpha}=\operatorname{span}\left\{F l_{0}^{\alpha}(x), F l_{1}^{\alpha}(x), \ldots, F l_{m-1}^{\alpha}(x)\right\}$. If $u_{m}(x)=\mathbf{C}^{T} \boldsymbol{\Phi}(x)$ is the best approximation to $u(x)$ from $\mathbf{P}_{m}^{\alpha}$, then the error bound is presented as follows:

$$
\left\|u(x)-u_{m}(x)\right\|_{\omega} \leq \frac{M_{\alpha}}{\Gamma(m \alpha+1)} \sqrt{\frac{1}{(2 m+1) \alpha}},
$$

where $M_{\alpha} \geq\left|D^{m \alpha} u(x)\right|, x \in[0,1]$. 
Proof. Consider the generalized Taylor's formula

$$
\begin{aligned}
& u(x) \\
& =\sum_{i=0}^{m-1} \frac{x^{i \alpha}}{\Gamma(i \alpha+1)} D^{i \alpha} u\left(0^{+}\right)+\frac{x^{m \alpha}}{\Gamma(m \alpha+1)} D^{m \alpha} u(\xi),
\end{aligned}
$$

where $0<\xi<x, x \in[0,1]$.

With Definition 4

$$
\left|u(x)-\sum_{i=0}^{m-1} \frac{x^{i \alpha}}{\Gamma(i \alpha+1)} D^{i \alpha} f\left(0^{+}\right)\right| \leq M_{\alpha} \frac{x^{m \alpha}}{\Gamma(m \alpha+1)} \text {. }
$$

Since $u_{m}(x)=\mathbf{C}^{T} \boldsymbol{\Phi}(x)$ is the best approximation to $u$ from $\mathbf{P}_{m}^{\alpha}$ and $\sum_{i=0}^{m-1}\left(x^{i \alpha} / \Gamma(i \alpha+1)\right) D^{i \alpha} f\left(0^{+}\right) \in \mathbf{P}_{m}^{\alpha}$, hence

$$
\begin{aligned}
& \left\|u(x)-u_{m}(x)\right\|_{\omega}^{2} \\
& \leq\left\|u(x)-\sum_{i=0}^{m-1} \frac{x^{i \alpha}}{\Gamma(i \alpha+1)} D^{i \alpha} u\left(0^{+}\right)\right\|_{\omega}^{2} \\
& \leq \frac{M_{\alpha}^{2}}{\Gamma(m \alpha+1)^{2}} \int_{0}^{1} x^{2 m \alpha} x^{\alpha-1} d x, \\
& \left\|u(x)-u_{m}(x)\right\|_{\omega}^{2} \leq \frac{M_{\alpha}^{2}}{\Gamma(m \alpha+1)^{2}} \int_{0}^{1} x^{(2 m+1) \alpha-1} d x \\
& =\frac{M_{\alpha}^{2}}{\Gamma(m \alpha+1)^{2}(2 m+1) \alpha} .
\end{aligned}
$$

$$
\begin{aligned}
& \int_{0}^{1} \int_{0}^{1} u(x, t) F l_{m}^{\alpha}(x) F l_{n}^{\beta}(t) \omega_{l}^{\alpha}(x) \omega_{l}^{\beta}(t) d x d t=\sum_{i=0}^{\infty} \sum_{j=0}^{\infty} u_{i j} \int_{0}^{1} \int_{0}^{1} F l_{i}^{\alpha}(x) F l_{j}^{\beta}(t) F l_{m}^{\alpha}(x) F l_{n}^{\beta}(t) \omega_{l}^{\alpha}(x) \omega_{l}^{\beta}(t) d x d t \\
& \quad=\sum_{i=0}^{\infty} \sum_{j=0}^{\infty} u_{i j} \int_{0}^{1} \omega_{l}^{\alpha}(x) F l_{i}^{\alpha}(x) F l_{m}^{\alpha}(x) d x \int_{0}^{1} \omega_{l}^{\beta}(t) F l_{i}^{\beta}(t) F l_{m}^{\alpha}(t) d t=u_{i j} \int_{0}^{1} \omega_{l}^{\alpha}(x)\left[F l_{i}^{\alpha}(x)\right]^{2} d x \int_{0}^{1} \omega_{l}^{\beta}(t)\left[F l_{i}^{\beta}(t)\right]^{2} d t \\
& \quad=u_{i j} \frac{1}{(2 i+1) \alpha} \frac{1}{(2 j+1) \beta} .
\end{aligned}
$$

Theorem 8. If the function $u(x, t)$ is a continuous function on $[0,1] \times[0,1]$ and the series $\sum_{i=0}^{\infty} \sum_{j=0}^{\infty} u_{i j} F l_{i}^{a}(x) F l_{j}^{\beta}(t)$ converges uniformly to $u(x, t)$, then $\sum_{i=0}^{\infty} \sum_{j=0}^{\infty} u_{i j} F l_{i}^{a}(x) F l_{j}^{\beta}(t)$ is the FLFs expansion of $u(x, t)$.

Proof. Using contradiction, let

$$
\begin{aligned}
& u(x, t)=\sum_{i=0}^{\infty} \sum_{j=0}^{\infty} u_{i j} F l_{i}^{a}(x) F l_{j}^{\beta}(t), \\
& u(x, t) \approx \sum_{i=0}^{\infty} \sum_{j=0}^{\infty} g_{i j} F l_{i}^{a}(x) F l_{j}^{\beta}(t) .
\end{aligned}
$$

Then there is at least one coefficient such that $u_{n m} \neq g_{n m}$; however

$$
\begin{gathered}
u_{n m}=(2 m+1)(2 n+1) \alpha \beta \int_{0}^{1} \int_{0}^{1} u(x, t) F l_{m}^{a}(x) \\
\cdot F l_{n}^{\beta}(t) \omega_{l}^{\alpha}(x) \omega_{l}^{\beta}(t) d x d t=g_{n m} .
\end{gathered}
$$

Proof. By multiplying $\omega_{l}^{\alpha}(x) \omega_{l}^{\beta}(t) F l_{m}^{\alpha}(x) F l_{n}^{\beta}(t)$ on both sides of (25), where $i$ and $j$ are fixed and integrating term wise with regard to $x$ and $t$ on $[0,1] \times[0,1]$, then 


$$
\begin{aligned}
& u(x, t) \approx \sum_{i=0}^{\infty} \sum_{j=0}^{\infty} u_{i j} F l_{i}^{a}(x) F l_{j}^{\beta}(t), \\
& f(x, t) \approx \sum_{i=0}^{\infty} \sum_{j=0}^{\infty} u_{i j} F l_{i}^{a}(x) F l_{j}^{\beta}(t) .
\end{aligned}
$$

By subtracting the above two equations with each other, we have

$$
\begin{aligned}
u(x, t)-f(x, t) & \approx \sum_{i=0}^{\infty} \sum_{j=0}^{\infty}\left(u_{i j}-u_{i j}\right) F l_{i}^{a}(x) F l_{j}^{\beta}(t) \\
& =0=\sum_{i=0}^{\infty} \sum_{j=0}^{\infty} 0 F l_{i}^{a}(x) F l_{j}^{\beta}(t) .
\end{aligned}
$$

Theorem 10. If the sum of the absolute values of the FLFs coefficients of a continuous function $u(x, t)$ forms a convergent series, then the FLFs expansion is absolutely uniformly convergent and converges to the function $u(x, t)$.

Proof. Consider

$$
\begin{aligned}
& \left|\sum_{i=0}^{\infty} \sum_{j=0}^{\infty} u_{i j} F l_{i}^{a}(x) F l_{j}^{\beta}(t)\right| \leq \sum_{i=0}^{\infty} \sum_{j=0}^{\infty}\left|u_{i j}\right|\left|F l_{i}^{a}(x)\right|\left|F l_{j}^{\beta}(t)\right| \\
& \quad \leq \sum_{i=0}^{\infty} \sum_{j=0}^{\infty}\left|u_{i j}\right| .
\end{aligned}
$$

If we consider truncated series in (25), we obtain

$$
u(x, t) \approx \sum_{i=0}^{m-1} \sum_{j=0}^{n-1} u_{i j} F l_{i}^{a}(x) F l_{j}^{\beta}(t)=\Phi^{T}(x) \mathbf{U} \boldsymbol{\Phi}(t)
$$

where

$$
\begin{aligned}
\Phi(x) & =\left[F l_{0}^{\alpha}(x), F l_{1}^{\alpha}(x), \ldots, F l_{m-1}^{\alpha}(x)\right]^{T} ; \\
\Phi(t) & =\left[F l_{0}^{\beta}(t), F l_{1}^{\beta}(t), \ldots, F l_{n-1}^{\beta}(t)\right]^{T}, \\
\mathbf{U} & =\left\{u_{i j}\right\}_{i, j=0}^{m-1, n-1} .
\end{aligned}
$$

In this paper, we use the Tau method [20] to compute the coefficients $u_{i j}$.

\section{Fractional Differential Operational Matrix of FLFs}

The derivative of the $\Phi(x)$ can be approximated

$$
D^{\gamma} \Phi(x) \approx \mathbf{D}^{\gamma} \Phi(x),
$$

where $\mathbf{D}^{\gamma}$ is called the FLFs operational matrix of derivative.
Theorem 11. Suppose $\mathbf{D}^{\gamma}$ is the $m \times m$ operational matrix of Caputo fractional derivatives of order $\gamma>0, \alpha>\gamma / 2$, when $\alpha \notin \mathbb{N}$; then the elements of $\mathbf{D}^{\gamma}$ are obtained as

$$
\begin{aligned}
& \left\{d_{i j}\right\}_{i, j}^{m-1 ; m-1}=(2 j+1) \\
& \cdot \alpha \sum_{s=0}^{i} \sum_{r=0}^{j} b_{r, j} b_{s, i}^{\prime} \frac{\Gamma(s \alpha+1)}{\Gamma(s \alpha-\gamma+1)} \frac{1}{(s+r+1) \alpha-\gamma},
\end{aligned}
$$

where

$$
\begin{aligned}
& b_{s, i}^{\prime} \\
& = \begin{cases}0, & s \alpha \in N_{0}, \quad s \alpha<\gamma, \\
b_{s, i}^{\prime}=b_{s, i} & s \alpha \notin N_{0}, \quad s \alpha \geq[\gamma] \text { or } s \alpha \in N_{0}, s \alpha \geq \gamma .\end{cases}
\end{aligned}
$$

Proof. With the properties of the derivative (ii) and the orthogonally of FLFs, we have

$$
D^{\gamma} F l_{i}^{\alpha}(x)=\sum_{s=0}^{i} b_{s, i}^{\prime} \frac{\Gamma(s \alpha+1)}{\Gamma(s \alpha-\gamma+1)} x^{s \alpha-\gamma} .
$$

Let

$$
x^{s \alpha-\gamma}=\sum_{j=0}^{m-1} d_{j} F l_{j}^{\alpha}(x)
$$

Multiplying $\omega_{l}^{\alpha}(x) F l_{j}^{\alpha}(x)$ on both sides of (40), we have

$$
d_{j}=(2 j+1) \alpha \sum_{r=0}^{j} b_{r, j} \frac{1}{(s+r+1) \alpha-\gamma} .
$$

Substituting (40) and (41) into (39), we have

$$
\begin{aligned}
& D^{\gamma} F l_{i}^{\alpha}(x)=(2 j+1) \\
& \quad \alpha \sum_{j=0}^{m-1} \sum_{s=0}^{i} \sum_{r=0}^{j} \frac{b_{r, j} b_{s, i}^{\prime} \Gamma(s \alpha+1)}{\Gamma(s \alpha-\gamma+1)} \frac{1}{(s+r+1) \alpha-\gamma}
\end{aligned}
$$

$$
\cdot F l_{j}^{\alpha}(x) \text {. }
$$

Hence,

$$
\begin{aligned}
& d_{i j}=(2 j+1) \\
& \cdot \alpha \sum_{s=0}^{i} \sum_{r=0}^{j} b_{r, j} b_{s, i}^{\prime} \frac{\Gamma(s \alpha+1)}{\Gamma(s \alpha-\gamma+1)} \frac{1}{(s+r+1) \alpha-\gamma}, \\
& i, j=0,1, \ldots, m-1 .
\end{aligned}
$$

\section{Description of the Proposed Method}

We consider a class of fractional partial differential equations as follows:

$$
\frac{\partial^{v} u(x, t)}{\partial x^{v}}+\frac{\partial^{\gamma} u(x, t)}{\partial t^{\gamma}}=g(x, t)
$$


subject to the initial conditions

$$
u(0, t)=u(x, 0)=0,
$$

where $0 \leq x, t \leq 1, n-1<v, \gamma \leq n, \partial^{v} u(x, t) / \partial x^{v}$ and $\partial^{\gamma} u(x, t) / \partial t^{\gamma}$ are fractional derivative in Caputo sense, $g(x, t)$ is the known continuous function, and $u(x, t)$ is the unknown function.

If we approximate function $u(x, t)$ by a terms of the FLFs, it can be written as (34). Then we have

$$
\begin{aligned}
\frac{\partial^{v} u(x, t)}{\partial x^{v}} & \approx \frac{\partial^{v}\left(\boldsymbol{\Phi}^{T}(x) \mathbf{U} \boldsymbol{\Phi}(t)\right)}{\partial x^{v}} \\
& =\frac{\partial^{v}\left(\boldsymbol{\Phi}^{T}(x)\right)}{\partial x^{v}} \mathbf{U} \boldsymbol{\Phi}(t) \\
& =\left(\frac{\partial^{v} \boldsymbol{\Phi}(x)}{\partial x^{v}}\right)^{T} \mathbf{U} \boldsymbol{\Phi}(t) \\
& \approx \boldsymbol{\Phi}^{T}(x)\left(\mathbf{D}^{v}\right)^{T} \mathbf{U} \boldsymbol{\Phi}(t), \\
\frac{\partial^{\gamma} u(x, t)}{\partial t^{\gamma}} & \approx \frac{\partial^{\gamma}\left(\boldsymbol{\Phi}^{T}(x) \mathbf{U} \boldsymbol{\Phi}(t)\right)}{\partial t^{\gamma}}=\boldsymbol{\Phi}^{T}(x) \mathbf{U} \frac{\partial^{\gamma} \boldsymbol{\Phi}(t)}{\partial t^{\gamma}} \\
& \approx \boldsymbol{\Phi}^{T}(x) \mathbf{U} \mathbf{D}^{\gamma} \boldsymbol{\Phi}(t), \\
g(x, t) & \approx \boldsymbol{\Phi}^{T}(x) \mathbf{G} \boldsymbol{\Phi}(t),
\end{aligned}
$$

$$
\cdot \omega_{l}^{\alpha}(x) \omega_{l}^{\beta}(t) d x d t
$$

Substituting (46) into (44), we have

$$
\begin{gathered}
\boldsymbol{\Phi}^{T}(x)\left(\mathbf{D}^{v}\right)^{T} \mathbf{U} \boldsymbol{\Phi}(t)+\boldsymbol{\Phi}^{T}(x) \mathbf{U} \mathbf{D}^{\gamma} \boldsymbol{\Phi}(t) \\
\approx \boldsymbol{\Phi}^{T}(x) \mathbf{G} \boldsymbol{\Phi}(t) .
\end{gathered}
$$

Similar to the typical Tau method [20], then we generate $m n-n-m+1$ algebraic equations by multiplying $\omega_{l}^{\alpha}(x) \omega_{l}^{\beta}(t) F l_{i}^{\alpha}(x) F l_{j}^{\beta}(t)$ (for $i=0,1,2, \ldots, m-2 ; j=$ $0,1,2, \ldots, n-2)$, integrating from 0 tol and using the orthogonal property, we have

$$
\mathbf{H}=\left(\mathbf{D}^{v}\right)^{T} \mathbf{U}+\mathbf{U D}^{\gamma}-\mathbf{G}=0 .
$$

For condition. (45), we have

$$
\begin{gathered}
u(0, t) \approx \boldsymbol{\Phi}^{T}(0) \mathbf{U} \boldsymbol{\Phi}(t) \approx \mathbf{C}^{T} \boldsymbol{\Phi}(t), \\
u(x, 0) \approx \boldsymbol{\Phi}^{T}(x) \mathbf{U} \boldsymbol{\Phi}(0) \approx \boldsymbol{\Phi}^{T}(x) \mathbf{F},
\end{gathered}
$$

with

$$
\begin{aligned}
\mathbf{C}^{T} & =\left\{c_{i}\right\}_{i=0}^{n-1}, \\
c_{i} & =(2 i+1) \beta \int_{0}^{1} u(0, t) F l_{i}^{\beta}(t) \omega_{l}^{\beta}(t) d t, \\
\mathbf{F} & =\left\{f_{j}\right\}_{j=0}^{m-1}, \\
f_{j} & =(2 j+1) \alpha \int_{0}^{1} u(x, 0) F l_{j}^{\alpha}(x) \omega_{l}^{\alpha}(x) d x .
\end{aligned}
$$

So we obtain

$$
\begin{gathered}
\boldsymbol{\Phi}^{T}(0) \mathbf{U} \approx \mathbf{C}^{T} ; \\
\mathbf{U} \boldsymbol{\Phi}(0) \approx \mathbf{F} .
\end{gathered}
$$

Equations (49) and (52) generate $m n+1$ linear algebraic equations. By solving the linear algebraic equations, the unknown coefficients $u_{i j}$ can be obtained.

\section{Error Analysis}

We consider $e(x, t)=u(x, t)-u_{m n}(x, t)$ as the error function of the approximate solution $u_{m n}(x, t)$ for $u(x, t)$, where $u(x, t)$ is the exact solution of (44) [21].

Therefore, $u_{m n}(x, t)$ satisfies the following problem:

$$
\frac{\partial^{v} u_{m n}(x, t)}{\partial x^{v}}+\frac{\partial^{\gamma} u_{m n}(x, t)}{\partial t^{\gamma}}+R_{m n}(x, t)=g(x, t),
$$

where $R_{m n}(x, t)$ is the residual function,

$$
R_{m n}(x, t)=\frac{\partial^{v} u_{m n}(x, t)}{\partial x^{v}}+\frac{\partial^{\gamma} u_{m n}(x, t)}{\partial t^{\gamma}}-g(x, t) .
$$

We find an approximation $e_{m n}^{\prime}(x, t)$ to the error function $e_{m n}(x, t)$ in the same way as we did before for the solution of the problem, the error function satisfies the problem

$$
\frac{\partial^{v} e_{m n}(x, t)}{\partial x^{v}}+\frac{\partial^{\gamma} e_{m n}(x, t)}{\partial t^{\gamma}}=R_{m n}(x, t) .
$$

We should note that, in order to construct the approximate $e_{m n}^{\prime}(x, t)$ to $e_{m n}(x, t)$, only (55) needs to be recalculated in the same way as we did before for the solution of (44).

\section{Numerical Examples}

Example 1. We consider the following FPDE:

$$
\begin{aligned}
\frac{\partial^{1 / 4} u(x, t)}{\partial x^{1 / 4}}+\frac{\partial^{1 / 2} u(x, t)}{\partial t^{1 / 2}} & =g(x, t), \\
u(0, t) & =0, \\
u(x, 0) & =0,
\end{aligned}
$$




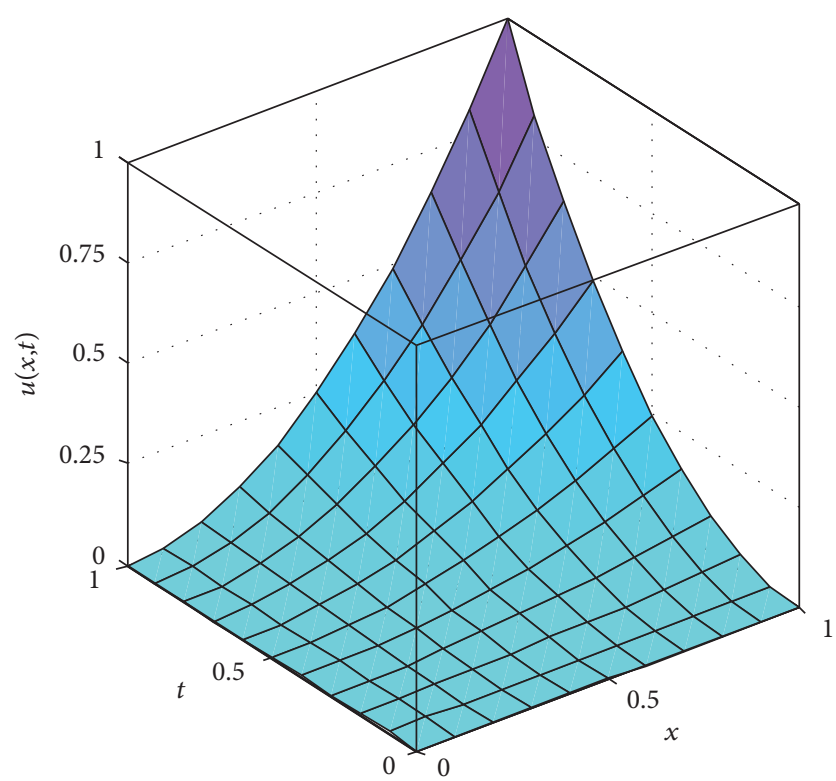

FIgURE 1: Numerical solution with $m=6$ for Example 1 .

where $g(x, t)=8 x^{2} t^{3 / 2} / 3 \sqrt{\pi}+32 t^{2} x^{7 / 4} / 21 \Gamma(3 / 4)$; using the method presented in Section 5 , the equation is transformed as follows:

$$
\begin{aligned}
\left(\mathbf{D}^{1 / 4}\right)^{T} \mathbf{U}+\mathbf{U D}^{1 / 2} & \approx \mathbf{G} \\
\boldsymbol{\Phi}^{T}(0) \mathbf{U} & \approx \mathbf{C}^{T} \\
\mathbf{U} \boldsymbol{\Phi}(0) & \approx \mathbf{F} .
\end{aligned}
$$

By solving (57), we can obtain the numerical solution of the problem.

When $n=6, m=6$, the matrix $\mathbf{U}$ is get as follows:

$$
\mathbf{U}=\left[\begin{array}{cccccc}
0.0198 & 0.0396 & 0.0282 & 0.0098 & 0.0014 & 0 \\
0.0486 & 0.0973 & 0.0694 & 0.0242 & 0.0034 & 0 \\
0.054 & 0.108 & 0.077 & 0.027 & 0.0038 & 0 \\
0.0412 & 0.0824 & 0.0588 & 0.0206 & 0.0029 & 0 \\
0.0221 & 0.0443 & 0.0316 & 0.0110 & 0.0015 & 0 \\
0.0062 & 0.0123 & 0.0087 & 0.0030 & 0 & 0
\end{array}\right]
$$

When $m=8, n=6$, the matrix $\mathbf{U}$ is get as follows:

$$
\mathbf{U}=\left[\begin{array}{cccccc}
0.0198 & 0.0396 & 0.0282 & 0.0099 & 0.0013 & 0 \\
0.0486 & 0.0971 & 0.0693 & 0.0241 & 0.0034 & 0 \\
0.0540 & 0.1079 & 0.0770 & 0.0269 & 0.0038 & 0 \\
0.0407 & 0.0814 & 0.0580 & 0.0203 & 0.0029 & 0 \\
0.0225 & 0.0449 & 0.0320 & 0.0112 & 0.0016 & 0 \\
0.0092 & 0.0183 & 0.0131 & 0.0046 & 0 & 0 \\
0.0027 & 0.0054 & 0.0038 & 0.0013 & 0 & 0 \\
0.0005 & 0.0009 & 0 & 0.0002 & 0 & 0
\end{array}\right] .
$$

When $n=6$, Figures 1,2 , and 3 show the numerical solution of (56) for $\alpha=1 / 4, \beta=1 / 2$ with $m=6, m=$

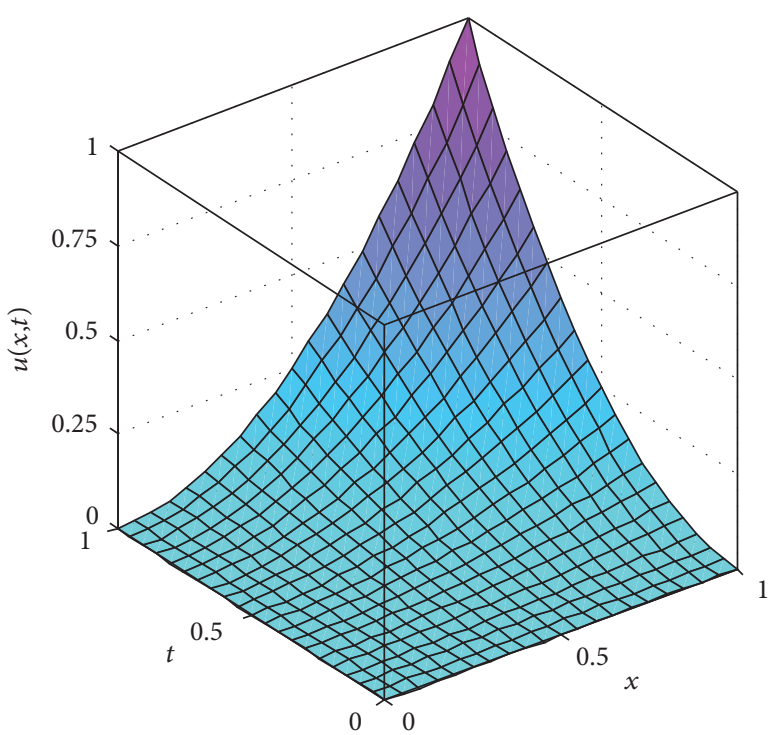

FIgURE 2: Numerical solution with $m=8$ for Example 1 .

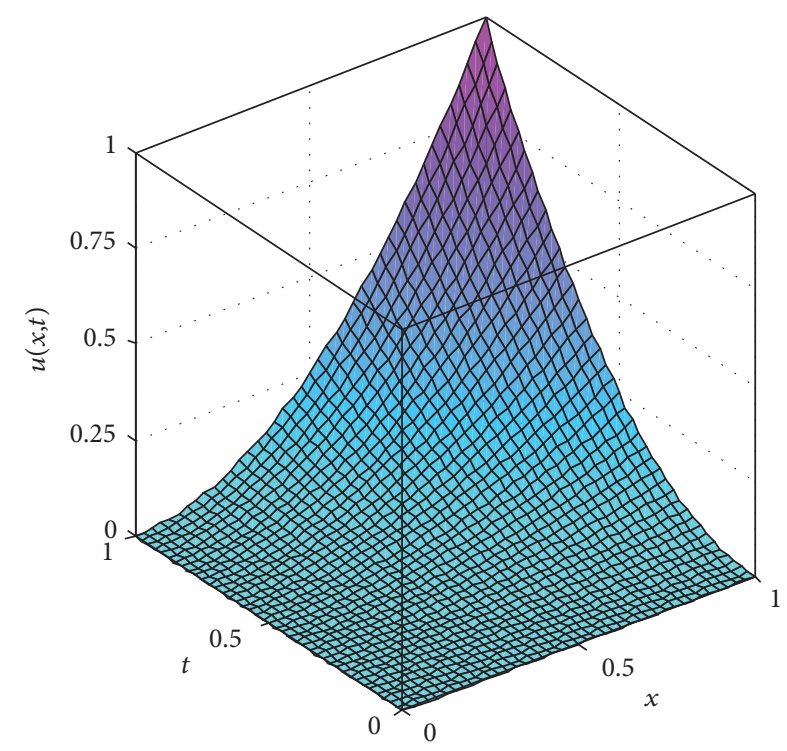

FIgURE 3: Numerical solution with $m=10$ for Example 1.

$8, m=10$. The exact solution is $x^{2} t^{2}$ which is shown in Figure 4 . We can see clearly that the numerical solutions are very good agreement with the exact solution. Particularly, when $m=10$ the numerical solution matches the exact solution very well.

Example 2. We consider the following equation:

$$
\begin{aligned}
\frac{\partial^{v} u(x, t)}{\partial x^{v}}+\frac{\partial^{\gamma} u(x, t)}{\partial t^{\gamma}} & =g(x, t), \\
u(0, t) & =0 .
\end{aligned}
$$




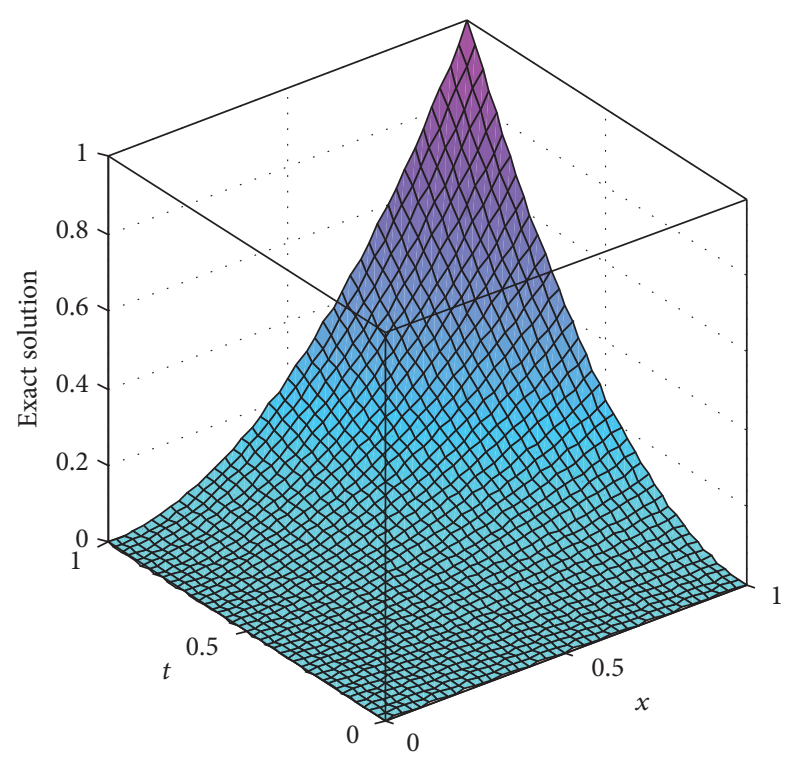

FIGURE 4: Exact solution for Example 1.

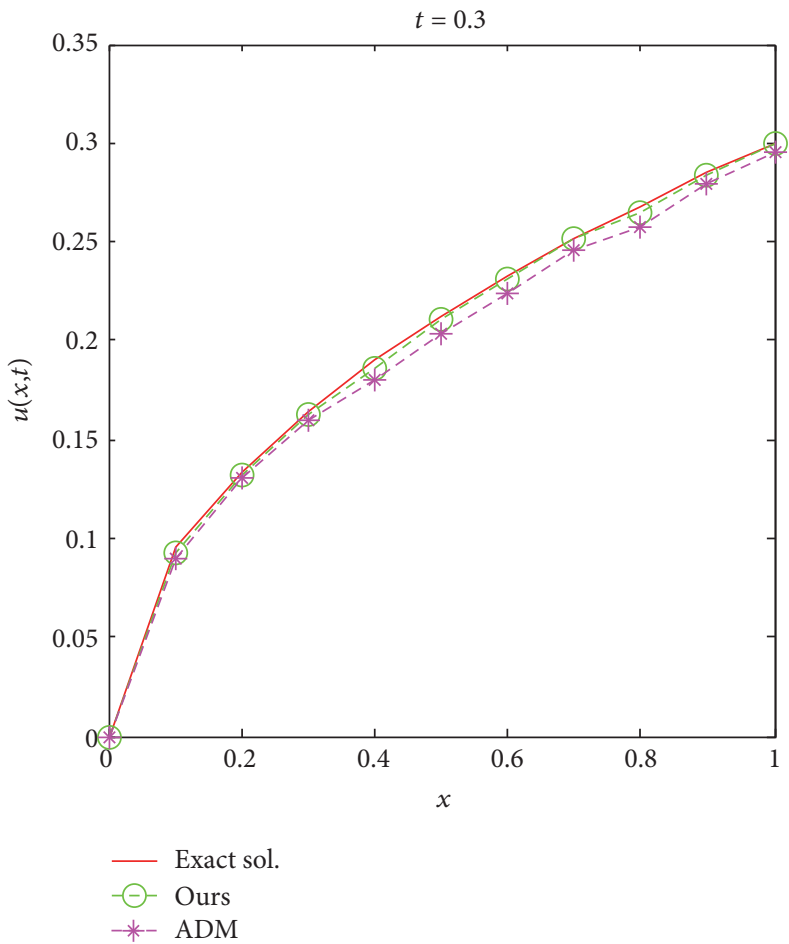

FIgURE 5: The comparison between FLFM and ADM with $m=3, n=3$ for Example 2.

The exact solution of this problem is $u(x, t)=x^{1 / 2} t$, where $v=\gamma=1 / 2, g(x, t)=\sqrt{\pi} t / 2+2 \sqrt{t x} / \sqrt{\pi}$. The equation is transformed as follows:

$$
\begin{aligned}
\left(\mathbf{D}^{1 / 2}\right)^{T} \mathbf{U}+\mathbf{U D}^{1 / 2} & \approx \mathbf{G} \\
\boldsymbol{\Phi}^{T}(0) \mathbf{U} & \approx \mathbf{C}^{T},
\end{aligned}
$$

when $m=3, n=3$, and $m=5, n=5$. The results obtained using our proposed method and ADM at $t=0.3$ are shown in Figures 5 and 6. Comparing the two figures, it can be concluded that the FLFM has a better advantage than ADM for solving fractional partial differential equations and the numerical results have achieved a good convergence precision when small $m$ and $n$ are selected. 


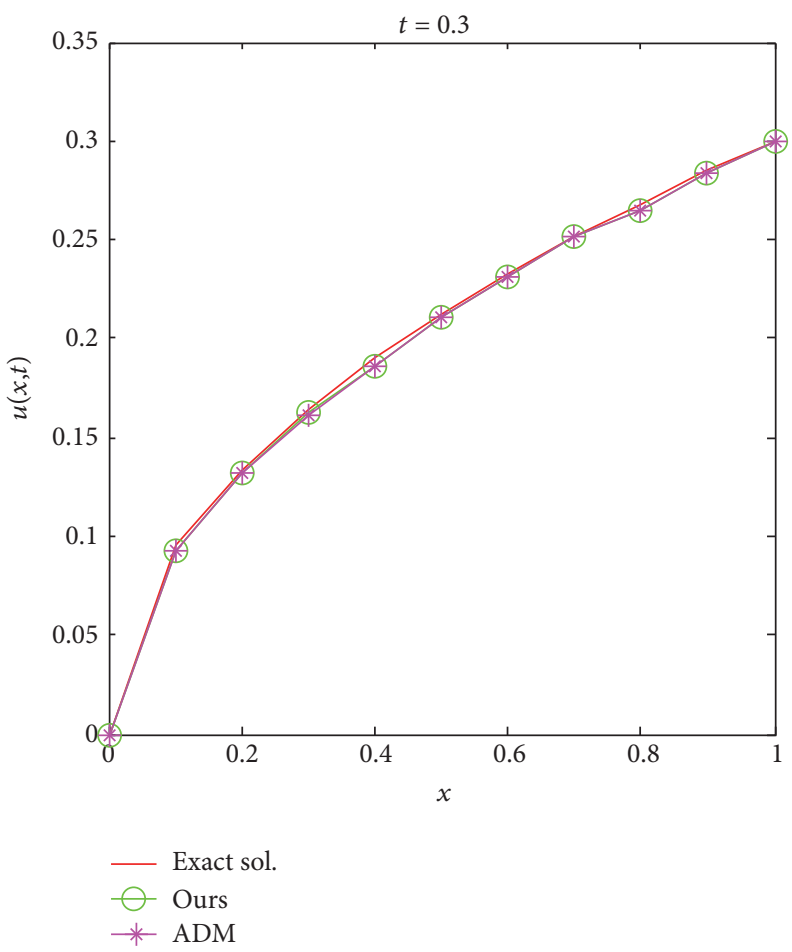

FIgURE 6: The comparison between FLFM and ADM with $m=5, n=5$ for Example 2.

Example 3. Consider the following fractional Poisson equation:

$$
\begin{aligned}
\frac{\partial^{v} u(x, t)}{\partial x^{v}}+\frac{\partial^{\gamma} u(x, t)}{\partial t^{\gamma}} & =g(x, t), \\
u(0, t) & =0, \\
u(x, 0) & =0 .
\end{aligned}
$$

(1) When $v=\gamma=5 / 4$,

$$
\begin{aligned}
g(x, t)= & \frac{5 \Gamma(1 / 4)}{16} x^{5 / 2}+\frac{5 \Gamma(1 / 4)}{16} t^{15 / 4} \\
& +\frac{30 \sqrt{\pi} x^{5 / 4} t^{5 / 4}}{16 \Gamma(9 / 4)}+\frac{2 t^{5 / 2} x^{5 / 4} \Gamma(15 / 4)}{\sqrt{\pi}} .
\end{aligned}
$$

The exact solution is $x^{5 / 2} t^{5 / 4}+x^{5 / 4} t^{15 / 4}$. When $n=4$, the absolute errors for $m=5, m=6, m=7$ are shown in Figures 7, 8, and 9.

(2) When $v=\gamma=1 / 2, g(x, t)=\sin (x+t)$. The exact solution is unknown.

Therefore, in order to show the efficiency of the present method, we define the error function as follows:

$$
e(x, t)=\boldsymbol{\Phi}^{T}(x)\left(\mathbf{D}^{v}\right)^{T} \mathbf{U} \boldsymbol{\Phi}(t)+\boldsymbol{\Phi}^{T}(x) \mathbf{U D}^{\gamma} \boldsymbol{\Phi}(t)
$$

$$
-\boldsymbol{\Phi}^{T}(x) \mathbf{G} \boldsymbol{\Phi}(t)
$$

Let $\alpha=\beta=1 / 2$ with $m=n=8, m=n=9$, and $m=n=10$; the absolute error $|e(x, t)|$ is shown in Figures 10, 11 , and 12 .

When $m=n=7$, we can have the coefficients matrix $\mathbf{U}$.

$$
\mathbf{U}=\left[\begin{array}{ccccccc}
0.1570 & 0.1708 & 0.0198 & 0 & 0.0060 & 0 & 0 \\
0.1708 & 0.1482 & -0.0152 & 0 & -0.0051 & -0.0001 & 0.0002 \\
0.0198 & -0.0153 & -0.0381 & -0.0007 & 0.0019 & -0.0004 & 0.0001 \\
-0.0003 & 0.0025 & -0.0007 & -0.0031 & 0.0006 & 0.0001 & -0.0001 \\
-0.0061 & -0.0051 & 0.0018 & 0.0006 & -0.0003 & 0.0001 & 0.0001 \\
0 & -0.0002 & -0.0003 & 0.0001 & 0.0001 & -0.0001 & -3.029 \\
-0.0002 & 0.0002 & 0.0001 & 0 & 0.0001 & -3.029 & 0
\end{array}\right]
$$

Then we have the numerical solution $u(x, t)=\Phi^{T}(x) \mathbf{U} \Phi(t)$. 


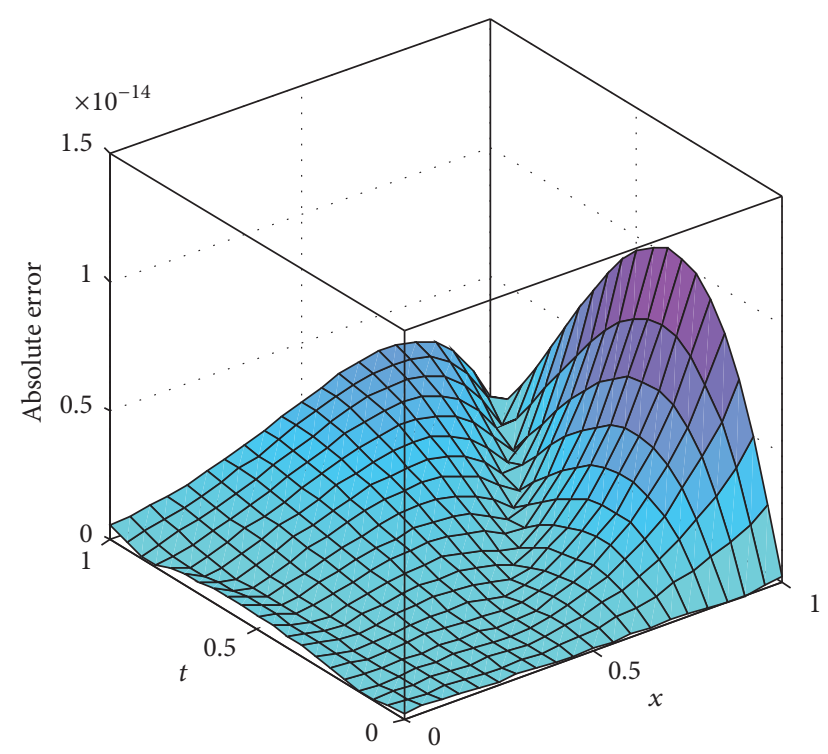

FIgURE 7: The absolute error for Example 3 with $m=5$.

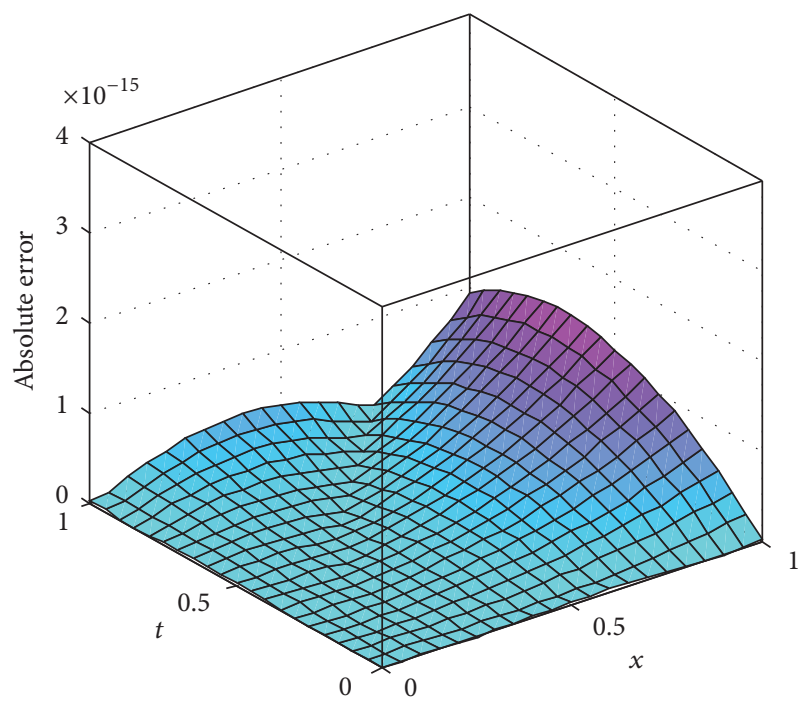

FIgURE 8: The absolute error for Example 3 with $m=6$.

\section{Conclusions}

In this paper, we define a basis of FLFs and have derived its fractional differential operational matrix. The fractional derivatives are described in the Caputo sense. The matrix together with Tau method is used to effectively approximate the exact solution of fractional partial differential equations. Compared with other numerical approach, the FLFs-Tau method can accurately represent properties of fractional calculus. Moreover, only a small number of FLFs are needed to obtain a satisfactory result. Numerical results which are given in the previous section demonstrate good accuracy of the algorithm. Finally, error analysis shows that the algorithm is convergent.

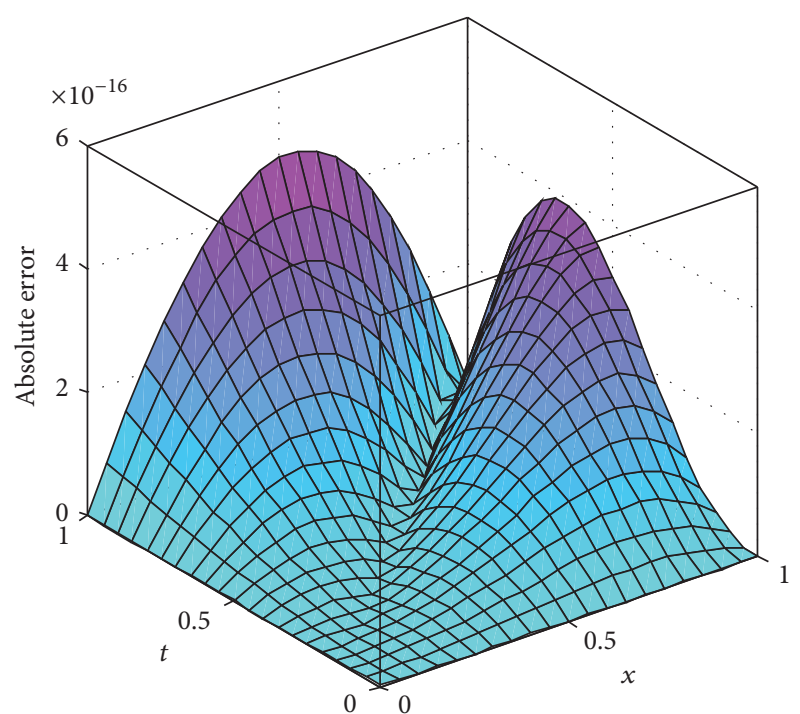

Figure 9: The absolute error for Example 3 with $m=7$.

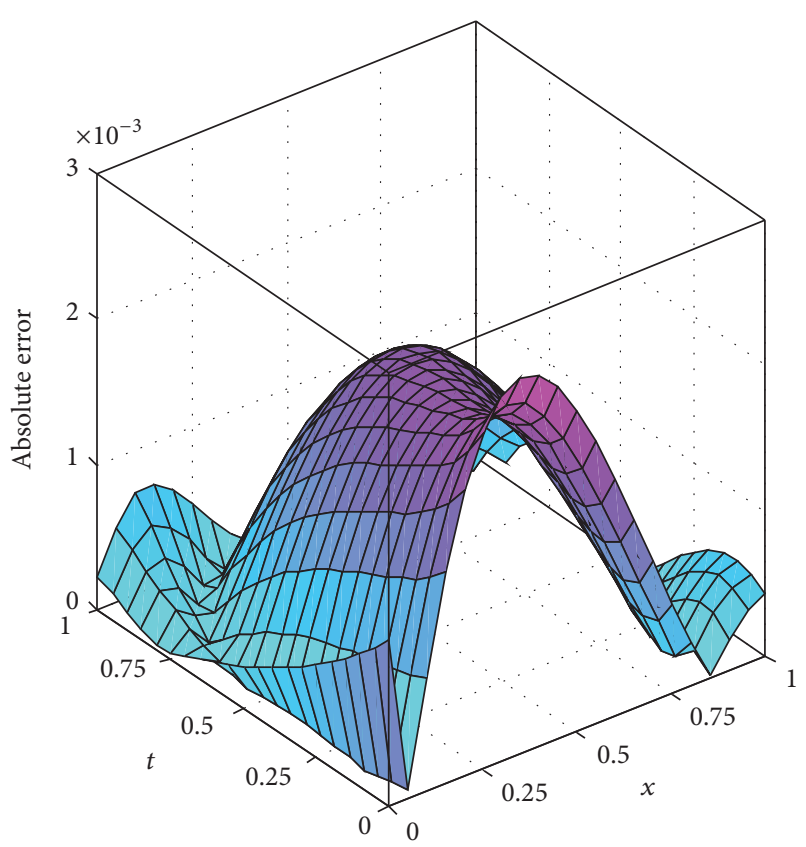

FIGURE 10: The absolute error for Example 3 with $m=n=8$.

\section{Competing Interests}

The authors declare that there is no conflict of interests regarding the publication of this paper.

\section{Acknowledgments}

This work was supported by Dr. Startup funds of Taiyuan University of Science and Technology (20122054). 


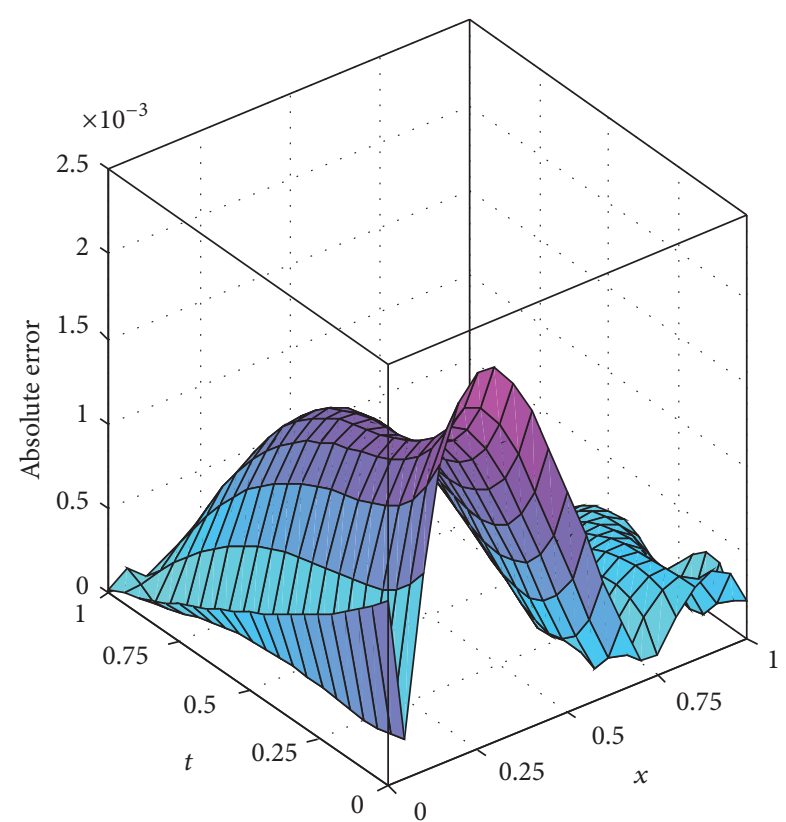

FIgURE 11: The absolute error for Example 3 with $m=n=9$.

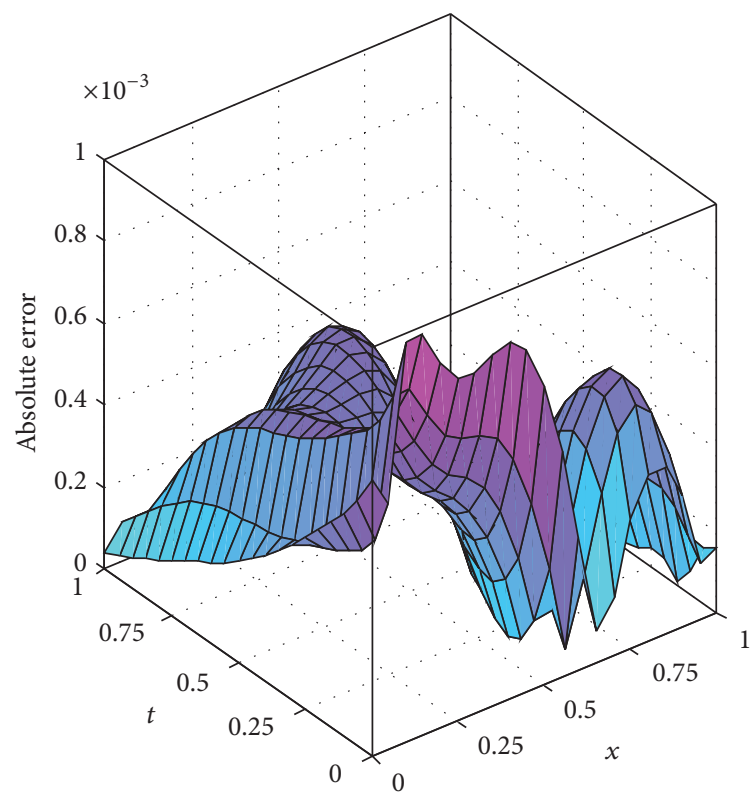

Figure 12: The absolute error for Example 3 with $m=n=10$.

\section{References}

[1] N. Liu and E.-B. Lin, "Legendre wavelet method for numerical solutions of partial differential equations," Numerical Methods for Partial Differential Equations, vol. 26, no. 1, pp. 81-94, 2010.

[2] Ü. Lepik, "Solving PDEs with the aid of two-dimensional Haar wavelets," Computers \& Mathematics with Applications, vol. 61, no. 7, pp. 1873-1879, 2011.

[3] S. Momani, Z. Odibat, and V. S. Erturk, "Generalized differential transform method for solving a space- and time-fractional diffusion-wave equation," Physics Letters. A, vol. 370, no. 5-6, pp. 379-387, 2007.
[4] Z. Odibat and S. Momani, "A generalized differential transform method for linear partial differential equations of fractional order," Applied Mathematics Letters, vol. 21, no. 2, pp. 194-199, 2008.

[5] W.-C. Tien and C.-K. Chen, "Adomian decomposition method by Legendre polynomials," Chaos, Solitons and Fractals, vol. 39, no. 5, pp. 2093-2101, 2009.

[6] Y. Zhang, "A finite difference method for fractional partial differential equation," Applied Mathematics and Computation, vol. 215, no. 2, pp. 524-529, 2009.

[7] Y. Chen, Y. Wu, Y. Cui, Z. Wang, and D. Jin, "Wavelet method for a class of fractional convection-diffusion equation with variable coefficients," Journal of Computational Science, vol. 1, no. 3, pp. 146-149, 2010.

[8] S. G. Venkatesh, S. K. Ayyaswamy, and S. R. Balachandar, "The Legendre wavelet method for solving initial value problems of Bratu-type," Computers \& Mathematics with Applications, vol. 63, no. 8, pp. 1287-1295, 2012.

[9] M. Yi and Y. Chen, "Haar wavelet operational matrix method for solving fractional partial differential equations," CMES. Computer Modeling in Engineering \& Sciences, vol. 88, no. 3, pp. 229-243, 2012.

[10] J. L. Wu, "A wavelet operational method for solving fractional partial differential equations numerically," Applied Mathematics and Computation, vol. 214, no. 1, pp. 31-40, 2009.

[11] F. Mohammadi and M. M. Hosseini, "A new Legendre wavelet operational matrix of derivative and its applications in solving the singular ordinary differential equations," Journal of the Franklin Institute. Engineering and Applied Mathematics, vol. 348, no. 8, pp. 1787-1796, 2011.

[12] Y. Li and N. Sun, "Numerical solution of fractional differential equations using the generalized block pulse operational matrix," Computers \& Mathematics with Applications, vol. 62, no. 3, pp. 1046-1054, 2011.

[13] Z. Odibat, "On Legendre polynomial approximation with the VIM or HAM for numerical treatment of nonlinear fractional differential equations," Journal of Computational and Applied Mathematics, vol. 235, no. 9, pp. 2956-2968, 2011.

[14] Y. Chen, M. Yi, C. Chen, and C. Yu, "Bernstein polynomials method for fractional convection-diffusion equation with variable coefficients," CMES. Computer Modeling in Engineering \& Sciences, vol. 83, no. 6, pp. 639-653, 2012.

[15] S. Kazem, "An integral operational matrix based on Jacobi polynomials for solving fractional-order differential equations," Applied Mathematical Modelling, vol. 37, no. 3, pp. 1126-1136, 2013.

[16] J. X. Wei, Y. M. Chen, and B. F. Li, "Numerical solution of spacetime fractional convection-diffusion equations with variable coefficients using haar wavelets," CMES: Computer Modeling in Engineering \& Sciences, vol. 89, no. 6, pp. 481-195, 2012.

[17] S. Z. Rida and A. M. Yousef, "On the fractional order Rodrigues formula for the Legendre polynomials," Advances and Applications in Mathematical Sciences, vol. 10, no. 5, pp. 509-517, 2011.

[18] M. Klimek and O. P. Agrawal, "Fractional Sturm-Liouville problem," Computers \& Mathematics with Applications, vol. 66, no. 5, pp. 795-812, 2013.

[19] S. Kazem, S. Abbasbandy, and S. Kumar, "Fractional-order Legendre functions for solving fractional-order differential equations," Applied Mathematical Modelling, vol. 37, no. 7, pp. 5498-5510, 2013. 
[20] M. Dehghan and A. Saadatmandi, "A tau method for the onedimensional parabolic inverse problem subject to temperature overspecification," Computers \& Mathematics with Applications, vol. 52, no. 6-7, pp. 933-940, 2006.

[21] Y. Chen, Y. Sun, and L. Liu, "Numerical solution of fractional partial differential equations with variable coefficients using generalized fractional-order Legendre functions," Applied Mathematics and Computation, vol. 244, pp. 847-858, 2014. 


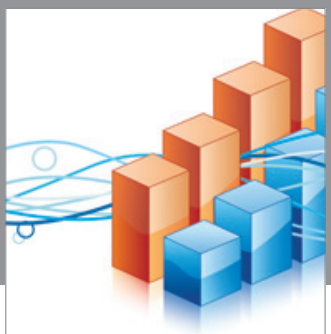

Advances in

Operations Research

vatem alat4

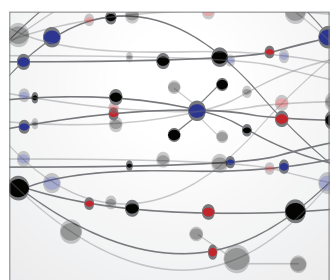

\section{The Scientific} World Journal
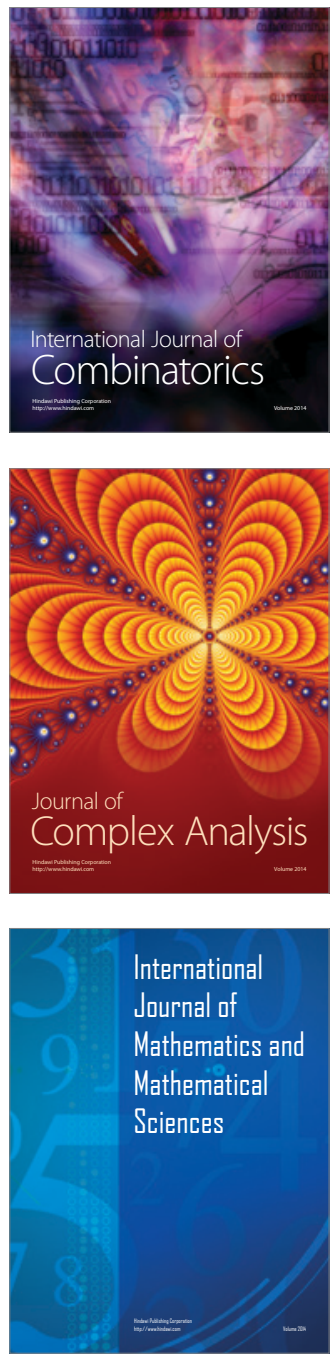
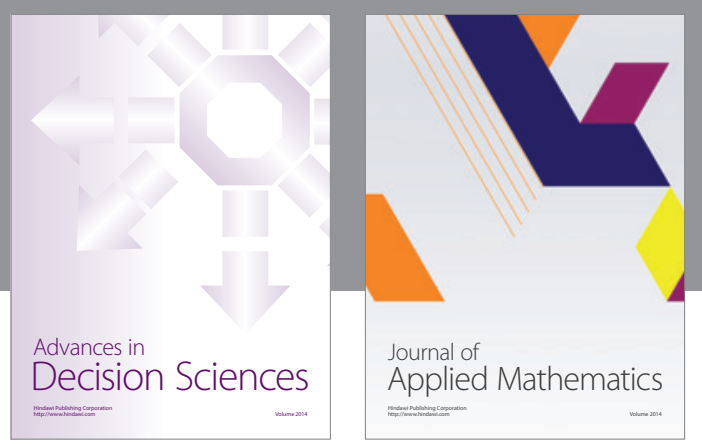

Algebra

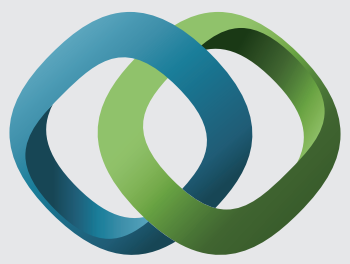

\section{Hindawi}

Submit your manuscripts at

https://www.hindawi.com
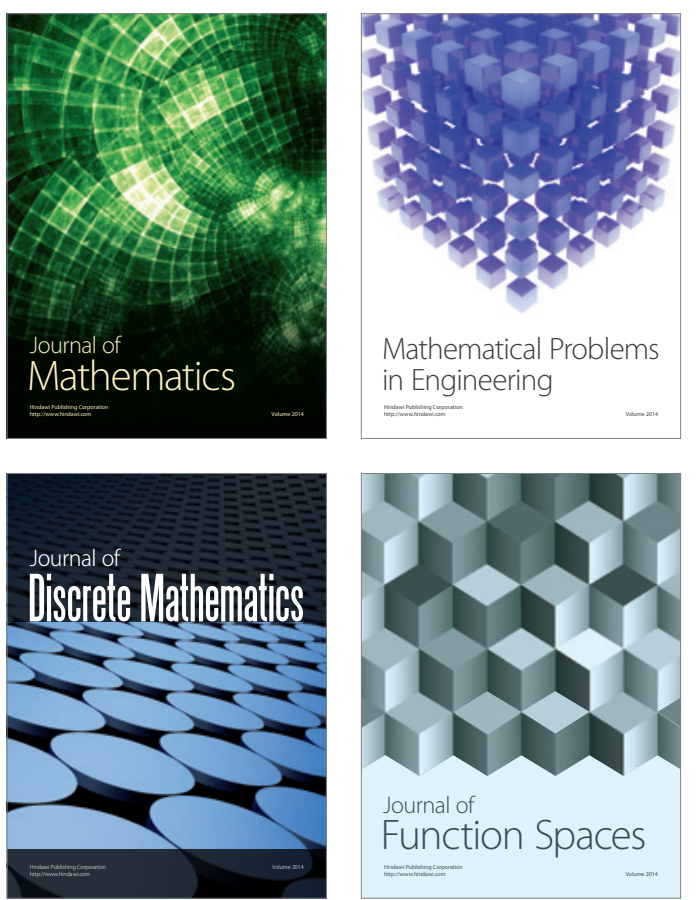

Mathematical Problems in Engineering
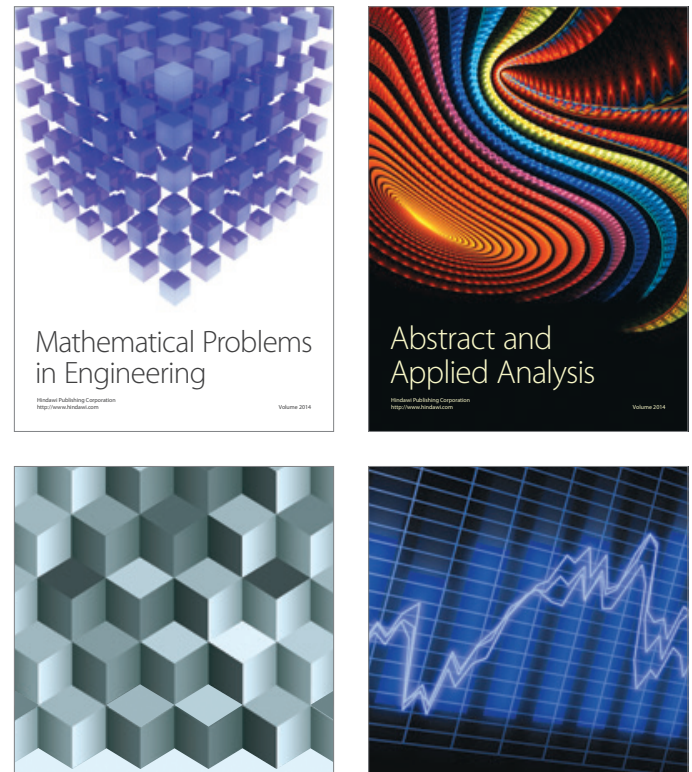

Journal of

Function Spaces

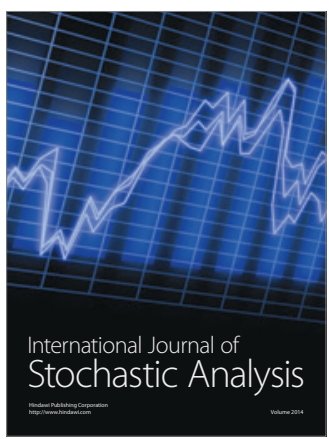

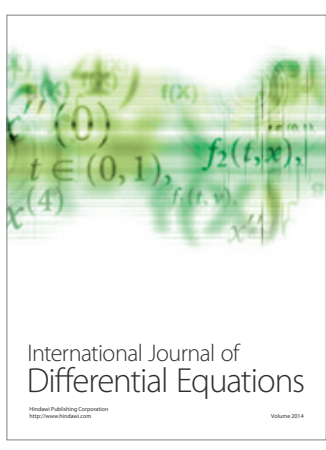
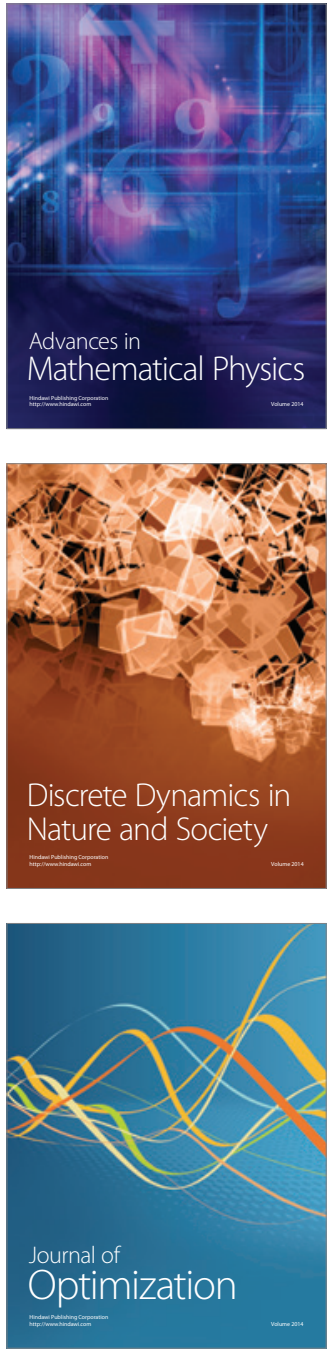